\title{
ANÁLISE DE DESEMPENHO DE CENTRO DE ESPECIALIDADES ODONTOLÓGICAS REGIONAL NO CEARÁ, BRASIL
}

\author{
PERFORMANCE ANALYSIS REGIONAL SPECIALIZED DENTAL \\ CARE IN THE STATE OF CEARÁ, BRAZIL
}

\section{Paulo Leonardo Ponte Marques', Antonio Rodrigues Ferreira Junior², Raiane Souza Saraiva ${ }^{3}$, Sarenne Pacheco Barbosa Carioca ${ }^{3}$, Lucianna Leite Pequeno ${ }^{4}$, Luiza Jane Eyre de Souza Vieira5.}

\section{RESUMO}

Objetivou-se avaliar o cumprimento das metas propostas pela Portaria GM n 1.464/2011 e Contrato de Programa Consorcial em um Centro de Especialidades Odontológicas Regional (CEO-R). Pesquisa quantitativa, descritiva e de corte transversal realizada no CEO-R Baturité, Ceará, Brasil. A coleta de dados foi conduzida por meio de dados secundários do Departamento de Informática do SUS e dos Relatórios do Sistema de Regulação Estadual, considerando 2014. Constatou-se que foram cumpridas as metas propostas pelo Ministério da Saúde e contrato consorcial, com exceção de procedimentos em pacientes especiais e ortodontia. O CEO-R não cumpriu a totalidade de metas previstas pelo Ministério da Saúde e no contrato de programa do consórcio público de saúde, estando sujeito à suspensão dos repasses de recursos financeiros federais e consorciais. A avaliação dos serviços subsidia o processo de tomada de decisão e pode reorientar o planejamento da oferta de vagas na Rede de Atenção à Saúde Bucal.

Descritores: Avaliação de Serviços de Saúde; Especialidades Odontológicas; Serviços de Saúde Bucal.

\section{ABSTRACT}

This study aimed to evaluate the fulfillment of the goals proposed by Ordinance GM No 1.464/2011 and the consortium Program Agreement on Regional Specialized Dental Care. Quantitative, descriptive and cross-sectional research on Regional Specialized Dental Care Baturité, Ceará, Brazil. Data collection was conducted through secondary data from the Department of Computer DATASUS and the State Regulation System Reports - UNISUWEB, considering 2014. It was found that the goals proposed by the Ministry of health and consortium Program Agreement were fulfilled, except for procedures in special patients and orthodontics targets. The Regional Specialized Dental Care has not fulfilled all targets set in Ministry of Health and the contract consortium program, subject to the suspension of transfers of federal financial resources and consorciais. The evaluation of services subsidizes the decision-making process and can reorient the planning of vacancies in Oral Health Dental Care.

Descriptors: Evaluation of Health services; Dental Specialty; Oral health services.
${ }^{1}$ Mestre em Saúde Coletiva pela Universidade de Fortaleza (UNIFOR), Fortaleza, CE, Brasil.

${ }^{2}$ Doutor em Saúde Coletiva pela Universidade Estadual de Campinas (UNICAMP), Campinas, SP, Brasil. ${ }^{1} \mathrm{M}$ pela Universidade $\mathrm{xxxx}$ (SIGLA), Cidade, Estado, Brasil.

${ }^{3}$ Discente da graduação em Odontologia pela Universidade de Fortaleza (UNIFOR), Fortaleza, CE, Brasil.

${ }^{4}$ Mestre em Saúde Pública pela Universidade Estadual do Ceará (UECE), Fortaleza, CE, Brasil.

${ }^{5}$ Doutora em Enfermagem pela Universidade Federal do Ceará (UFC), Fortaleza, CE, Brasil. 


\section{Introdução}

A Política Nacional de Saúde Bucal (PNSB), denominada Brasil Sorridente, foi implantada em 2004 pelo Ministério da Saúde (MS) com o propósito de desenvolver ações voltadas para a promoção da saúde, prevenção de agravos, diagnóstico, tratamento e reabilitação1. Isso exigiu mudanças nas ações de saúde bucal no Sistema Único de Saúde (SUS), as quais privilegiavam procedimentos clínicos cirúrgicos mutiladores.

Para isso, foram recomendadas diretrizes com vistas à reorganização da atenção primária em saúde bucal, com implantação de Equipes de Saúde Bucal na Estratégia de Saúde da Família (ESF); ampliação e qualificação da atenção secundária; assistência à saúde terciária; promoção e prevenção; vigilância; avaliação e monitoramento. A incorporação dessas diretrizes representou um grande impulso na melhoria da oferta de atendimento odontológico e atenção à saúde bucal $^{2-3}$

Em relação à qualificação e ampliação da assistência a atenção secundária, foi proposta a implantação de Centros de Especialidades Odontológicas (CEO), estabelecimentos de saúde classificados como clínica especializada ou ambulatório de especialidade, que ofertam, minimamente, serviços nas especialidades: diagnóstico bucal, periodontia especializada, cirurgia oral menor dos tecidos moles e duros, endodontia, atendimento a pacientes especiais ${ }^{4}$. Embora não estejam entre as especialidades mínimas, o MS também financia procedimentos ortodônticos e próteses dentárias para os municípios que implantam esses serviços ${ }^{5}$.

A implantação e o custeio dos CEO são realizados por meio de parcerias entre Municípios, Estados e o Governo Federal ${ }^{6}$, sendo este último responsável pelo repasse de recursos na forma de incentivos com variação de acordo com o porte do CEO e alcance das metas estabelecidas. Esses centros estão classificados em Tipo I, II e III: o tipo I dispõe de três cadeiras odontológicas; o tipo II de quatro a seis cadeiras odontológicas; e o tipo III com sete ou mais cadeiras odontológicas.

Em relação às metas nacionais, a Portaria GM No 1.464, de 24 de Junho de 2011 estabelece uma produção mínima de procedimentos a serem alcançados nas especialidades. Caso estas não sejam atingidas por dois meses consecutivos, ou três meses alternados, durante o período de um ano, os repasses de incentivos financeiros federais poderão ser suspensos, e somente retornarão após a regularização das metas mínimas exigidas ${ }^{7}$.

O Ceará entrou para história em 2004 quando o presidente da República inaugurou o primeiro CEO no Brasil Sorridente, garantindo prosseguimento à construção de novas unidades. Entre os anos de 2009 a 2014, o Estado ampliou sua rede de atenção secundária investindo cerca de $\mathrm{R} \$ 14$ milhões de reais para estruturação de CEO com características regionais, visando a integralidade e garantindo 0 acesso da população aos serviços odontológicos de média complexidade ${ }^{1,8}$.

Os CEO Regionais (CEO-R) fazem parte da gestão estadual, porém são administrados via gestão indireta por meio de Consórcio Público de Saúde (CPS), os quais constituem associações entre entes da federação com a finalidade de prestar serviços e ações conjuntas de interesse coletivo ${ }^{8}$. Esses centros devem cumprir as metas de oferta de vagas, conforme critérios populacionais, propostas no Contrato de Programa Consorcial, instrumento de gestão que indica 0 cumprimento de obrigações e oferta de vagas aos municípios consorciados.

Nesse contexto, a avaliação é uma ferramenta de gestão que possibilita analisar a efetividade da atenção secundária na rede de atenção à saúde, assim como levantar questionamentos sobre funcionamento dos serviços, utilização de vagas, acessibilidade e a relação com outros níveis de atenção, especialmente na promoção do acesso a cuidados odontológicos ${ }^{9-10}$.

Diante do exposto, este estudo objetivou avaliar o cumprimento das metas de procedimentos ambulatoriais propostas pela Portaria GM No 1.464/2011 e de metas de ofertas de vagas propostas pelo Contrato de Programa Consorcial de um CEO-R. 


\section{Metodologia}

Estudo quantitativo, descritivo, de caráter avaliativo e corte transversal. Realizado no estado do Ceará, que dispõe de 22 CEO-R, inaugurados a partir de 2009. Essas unidades integram o Programa de Expansão e Melhoria da Assistência Especializada à Saúde do Estado do Ceará, desenvolvido pela Secretaria Estadual de Saúde ${ }^{8}$.

O estudo focou o CEO-R de Baturité-CE, selecionado por estar na primeira região de saúde implantada no Estado, representando, portanto, o início do processo de regionalização. A unidade oferece serviços odontológicos especializados à população do maciço de Baturité, cuja região possui um total de 134 mil habitantes e é composta por oito municípios.

Os serviços especializados oferecidos no CEO-R são: Atendimento à Pacientes com Necessidades Especiais (PNE) (01 consultório), Cirurgia oral menor/Estomatologia (01 consultório), Endodontia (03 consultórios), Ortodontia (03 consultórios), Periodontia (01 consultório) e Prótese Dentária/Distúrbios da Articulação Têmporo-Mandibular (02 consultórios), atendendo pacientes referenciados a partir das equipes de saúde bucal da ESF dos municípios que fazem parte da Regional de Saúde.

Foram utilizados dados secundários originados a partir das bases de dados disponíveis no Departamento de Informática do SUS (DATASUS) e no Sistema de Regulação Estadual - UNISUSWEB. A coleta foi realizada em junho de 2015 em dois momentos, sendo o primeiro para captação dos procedimentos ambulatoriais realizados nas especialidades, seguindo-se um protocolo de coleta de dados (FIGURA 1) e o segundo para oferta de vagas.

Figura 1. Protocolo de coleta de dados da pesquisa realizado no site do Departamento de Informática do SUS (DATASUS).

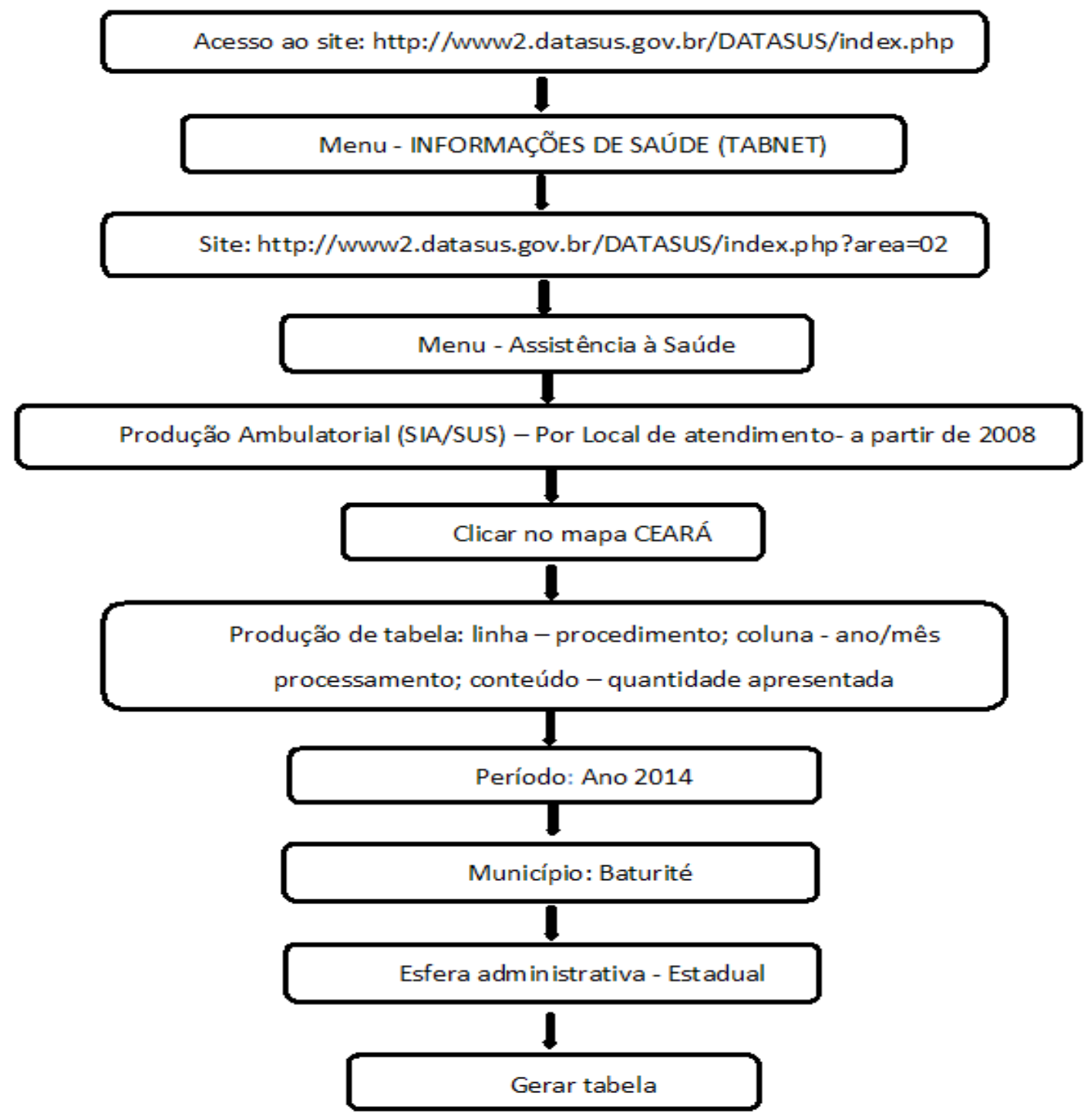


Os dados coletados foram avaliados conforme as metas propostas pela Portaria GM № 1.464, de Junho de 2011, dispostas na Tabela 1.

Tabela 1. Especialidades e metas propostas pela Portaria Nº 1.464 de Junho de 2011.

\begin{tabular}{|c|c|}
\hline Procedimentos conforme Especialidades & Metas para o CEO tipo III \\
\hline $\begin{array}{c}\text { Procedimentos básicos realizados em pessoas } \\
\text { com necessidades especiais }\end{array}$ & 190 \\
\hline $\begin{array}{c}\text { Procedimentos restauradores realizados em } \\
\text { pessoas com necessidades especiais }\end{array}$ & 95 \\
\hline Procedimentos de periodontia & 150 \\
\hline Procedimentos de endodontia & 95 \\
\hline $\begin{array}{c}\text { Procedimentos de endodontia em dentes } \\
\text { permanentes com 3 ou mais raízes }\end{array}$ & 19 \\
\hline Procedimentos de cirurgia oral & 170 \\
\hline
\end{tabular}

Fonte: BRASIL, 2011

Os dados de oferta de vagas foram coletados diretamente no CEO-R Baturité, por meio dos Relatórios de ofertas de vagas do Sistema de Regulação Estadual - UNISUSWEB, com anuência da Direção Geral da unidade para cada uma das especialidades ofertadas, e foram comparadas com as metas previstas no Contrato de Programa Consorcial, dispostas na Tabela 2.

Tabela 2. Metas mensais de oferta de vagas por especialidade no Contrato de Programa do CEO-R Baturité-CE, 2014.

\begin{tabular}{|c|c|}
\hline Especialidade & Metas \\
\hline $\begin{array}{c}\text { Oferta de vagas para Atendimento à } \\
\text { Pacientes com Necessidades Especiais }\end{array}$ & 48 \\
\hline Oferta de vagas para Endodontia & 176 \\
\hline $\begin{array}{c}\text { Oferta de vagas para Prótese Dentária/ } \\
\text { Disfunção Têmporo-Mandibular }\end{array}$ & 70 \\
\hline Oferta de vagas para Periodontia & 88 \\
\hline Oferta de vagas para Ortodontia & 88 \\
\hline $\begin{array}{c}\text { Oferta de vagas para Cirurgia Oral Menor/ } \\
\text { Estomatologia }\end{array}$ & 82 \\
\hline
\end{tabular}

A análise estatística foi realizada com base em medidas descritivas, utilizando-se a média como medida de tendência central e o desvio padrão (DP) como medida de dispersão. A média calculada foi obtida diante do somatório de toda a produção, dividida por 12, referente ao número de meses do ano.

Salienta-se que a pesquisa foi conduzida respeitando os padrões éticos vigentes.

\section{Resultados e Discussão}

De acordo com as metas propostas pela Portaria GM N01.464 de Junho de 2011, os dados da produção ambulatorial apresentada do CEO-R de Baturité estão dispostos na Tabela 3. 
Tabela 3. Produção de procedimentos ambulatoriais mensais no CEO-R Baturité-CE, 2014.

\begin{tabular}{|c|c|c|c|c|c|c|c|c|c|c|c|c|c|}
\hline $\begin{array}{l}\text { ESPECIALIDADE/ } \\
\text { OFERTA DE VAGAS }\end{array}$ & $\begin{array}{l}\mathrm{J} \\
\mathrm{A} \\
\mathrm{N}\end{array}$ & $\begin{array}{l}F \\
E \\
V\end{array}$ & $\begin{array}{l}M \\
A \\
R\end{array}$ & $\begin{array}{l}A \\
B \\
R\end{array}$ & $\begin{array}{c}\mathrm{M} \\
\mathrm{A} \\
\mathrm{I}\end{array}$ & $\begin{array}{l}\mathrm{J} \\
\mathrm{U} \\
\mathrm{N}\end{array}$ & $\begin{array}{l}J \\
U \\
L\end{array}$ & $\begin{array}{l}\text { A } \\
\text { C } \\
\text { O }\end{array}$ & $\begin{array}{l}S \\
E \\
T\end{array}$ & $\begin{array}{l}\mathrm{O} \\
\mathrm{U} \\
\mathrm{T}\end{array}$ & $\begin{array}{l}\mathrm{N} \\
\mathrm{O} \\
\mathrm{V}\end{array}$ & $\begin{array}{l}D \\
E \\
Z\end{array}$ & $\begin{array}{l}\text { MÉDIA } \\
\pm D P\end{array}$ \\
\hline $\begin{array}{c}\text { Procedimentos } \\
\text { básicos realizados } \\
\text { em PNE }\end{array}$ & 320 & 321 & 257 & 251 & 457 & 0 & 866 & 280 & 1880 & 749 & 680 & 205 & $522 \pm 495$ \\
\hline $\begin{array}{l}\text { Procedimentos } \\
\text { restauradores } \\
\text { realizados em PNE }\end{array}$ & 82 & 68 & 50 & 52 & 124 & 0 & 170 & 59 & 40 & 107 & 93 & 55 & $75 \pm 44$ \\
\hline $\begin{array}{l}\text { Procedimentos de } \\
\text { Periodontia }\end{array}$ & 151 & 422 & 180 & 318 & 203 & 0 & 633 & 310 & 1802 & 506 & 549 & 185 & $438 \pm 467$ \\
\hline $\begin{array}{l}\text { Procedimentos de } \\
\text { Endodontia }\end{array}$ & 105 & 68 & 72 & 147 & 109 & 0 & 201 & 103 & 653 & 147 & 127 & 69 & $150 \pm 166$ \\
\hline $\begin{array}{l}\text { Procedimentos } \\
\text { de endodontia } \\
\text { em dentes } \\
\text { permanentes com } \\
3 \text { ou mais raízes }\end{array}$ & 41 & 41 & 38 & 45 & 48 & 0 & 78 & 47 & 53 & 33 & 46 & 41 & $42 \pm 17$ \\
\hline $\begin{array}{l}\text { Procedimentos de } \\
\text { cirurgia oral }\end{array}$ & 134 & 192 & 141 & 195 & 222 & 0 & 415 & 223 & 782 & 179 & 170 & 83 & $228 \pm 199$ \\
\hline
\end{tabular}

Fonte: DATASUS, 2015

Das seis metas avaliadas, somente a referente aos Procedimentos Restauradores em PNE não foi alcançada, obtendo $79 \%$. Contudo, as outras cinco obtiveram percentuais maiores que 100\%, apresentando como resultados: Procedimentos Básicos em PNE com 275\%, Periodontia 292\%, Cirurgia Oral 134\%, Endodontia 158\%, Endodontia de dentes permanentes com 3 ou mais raízes com $221 \%$. Foi observada uma grande diferença da quantidade de procedimentos realizados de um mês para o outro, resultando em um alto desvio padrão. No mês de Junho não foi observado registro de procedimentos.

A Tabela 4 apresenta os resultados mensais de vagas ofertadas, por especialidade, para o ano de 2014.

\begin{tabular}{|c|c|c|c|c|c|c|c|c|c|c|c|c|c|}
\hline $\begin{array}{c}\text { ESPECIALIDADE/ } \\
\text { OFERTA DE } \\
\text { VAGAS }\end{array}$ & $\begin{array}{l}\mathrm{J} \\
\mathrm{A} \\
\mathrm{N}\end{array}$ & $\begin{array}{l}F \\
E \\
V\end{array}$ & $\begin{array}{l}M \\
A \\
R\end{array}$ & $\begin{array}{l}A \\
B \\
R\end{array}$ & $\begin{array}{l}M \\
A \\
I\end{array}$ & $\begin{array}{l}\mathrm{J} \\
\mathrm{U} \\
\mathrm{N}\end{array}$ & $\begin{array}{l}\mathrm{J} \\
\mathrm{U} \\
\mathrm{L}\end{array}$ & $\begin{array}{l}\text { A } \\
\text { G } \\
O\end{array}$ & $\begin{array}{l}S \\
E \\
T\end{array}$ & $\begin{array}{l}O \\
U \\
T\end{array}$ & $\begin{array}{l}\mathrm{N} \\
\mathrm{O} \\
\mathrm{V}\end{array}$ & $\begin{array}{l}D \\
E \\
Z\end{array}$ & $\begin{array}{c}\text { MÉDIA } \\
\pm D P\end{array}$ \\
\hline Periodontia & 57 & 100 & 65 & 104 & 83 & 85 & 111 & 112 & 108 & 107 & 80 & 61 & $89 \pm 19$ \\
\hline Endodontia & 217 & 217 & 143 & 211 & 179 & 178 & 223 & 175 & 182 & 172 & 186 & 139 & $184 \pm 26$ \\
\hline Cirurgia & 138 & 137 & 91 & 128 & 173 & 109 & 184 & 151 & 165 & 123 & 99 & 132 & $132 \pm 30$ \\
\hline Ortodontia & 08 & 08 & 04 & 13 & 0 & 21 & 16 & 21 & 16 & 16 & 16 & 0 & $16 \pm 18$ \\
\hline Prótese & 134 & 134 & 103 & 117 & 133 & 92 & 116 & 113 & 144 & 129 & 139 & 21 & $115 \pm 33$ \\
\hline PNE & 49 & 49 & 38 & 34 & 58 & 50 & 72 & 36 & 32 & 65 & 56 & 36 & $48 \pm 12$ \\
\hline
\end{tabular}

Fonte: UNISUSWEB, 2015

Em relação ao contrato de programa consorcial observou-se que as especialidades de Periodontia 103\%, Endodontia 106\%, Cirurgia 155\%, Prótese 164\%, e PNE 102\% conseguiram alcançar as metas pactuadas. No entanto, a especialidade de Ortodontia não conseguiu resultado satisfatório, pois obteve somente $16 \%$ em relação a meta pactuada. Todavia, esta especialidade também obteve um alto desvio padrão em relação a sua média, indicando uma grande variação dos resultados ao longo dos meses. 
A compreensão do quadro de resultados frente à avaliação auxilia a gestão na tomada de decisões, no planejamento em saúde, na divulgação das ações integrativas, assim como a direciona para oferta de serviços de acordo com as necessidades de saúde bucal da população.

Este estudo observou que o desempenho nos procedimentos ambulatoriais e oferta de vagas nas especialidades não apresentou uniformidade. Entre as causas possíveis se encontram: a inconsistência nas informações decorrentes do registro de procedimentos, erros no processo de digitação e falha na transferência de arquivos do Sistema de Informação Ambulatorial (SIA-SUS). Para que isso seja minimizado aponta-se a necessidade de padronização dos registros, treinamento de profissionais e capacitação de gestores visando adequado preenchimento dos instrumentos nos sistemas de informação e a consequente utilização dos dados na análise e difusão das informações ${ }^{11}$.

Dentre as sete metas federais avaliadas observou-se que somente a de procedimentos restauradores em PNE não foi alcançada, possivelmente devido às condições de saúde bucal nas quais esses pacientes acessam o serviço no CEO, muitas vezes com necessidades cirúrgicas, periodontais, ou ainda, estes realizarem os procedimentos odontológicos na atenção primária ou serviços privados.

Pesquisas realizadas No Rio Grande do Sul e Amazonas observaram situação semelhante, com o não cumprimento das meta estipuladas ${ }^{4,12}$.

Estudo realizado no estado de Pernambuco reforça a ideia de que para resultados satisfatórios faz-se necessário ampliar as relações entre os entes federativos e estimular a criação de novos modelos de gestão, tais como consórcios de saúde entre municípios ${ }^{13}$.

$\mathrm{Na}$ especialidade de periodontia o comportamento dos resultados foi diferente. Isso pode ser em decorrência do processo de organização na rede de atenção, demanda na busca do serviço especializado e o encaminhamento para - CEO. A oferta de vagas e os procedimentos ambulatoriais foram satisfatórios, sendo este último alcançado com uma média bem acima do valor pactuado.

Essa situação difere do estudo realizado por Góes para avaliação do cumprimento da meta da Atenção Secundária na Região Nordeste, apresentando como resultado o índice de 54\% no não cumprimento de meta na especialidade de periodontia ${ }^{14}$. Estudo realizado na Bahia comparou as taxas de utilização das vagas ofertadas em quatro tipos de CEO e observou-se que em todos houve uma predominância de procedimentos de periodontia típicos da atenção básica, sendo, portanto, necessário uma regulação na atenção básica para que os pacientes não sejam encaminhados para a atenção secundária sem que haja necessidade 9 .

Estudos avaliando a especialidade de endodontia nos CEO em Minas Gerais e na Bahia constataram que a situação é preocupante, pois não houve o cumprimento das metas estabelecidas ${ }^{15-16}$. Justifica-se possivelmente esse resultado pela ação da atenção primária, onde dentes com comprometimento pulpar são submetidos à exodontia.

Diferentemente, o presente estudo aponta que na especialidade de Endodontia as metas foram cumpridas, tanto na oferta de vagas, quanto nos procedimentos ambulatoriais. Esse resultado pode ser justificado pela organização da rede de atenção com diálogo entre profissionais e pacientes sobre a possibilidade de manutenção do elemento dentário.

Pesquisa realizada na região do Cariri-CE mostra que na especialidade de cirurgia não foram cumpridas as metas federais, com exceção de um CEO municipal. Cabe discussão se o quantitativo de procedimentos cirúrgicos a serem realizados pode ter sido estabelecido acima da capacidade instalada para CEO tipo III, impossibilitando esses Centros de alcançarem a referida meta ${ }^{17}$.

Entre as especialidades ofertadas, a prótese obteve uma média de vagas satisfatória, bem superior ao previsto no Contrato de Programa. Embora não esteja pactuada enquanto meta de procedimentos na Portaria GM No 1.464/2011, segundo a Portaria GM n 2486 de 31/10/2012, a faixa de produção de próteses dentárias para o CEO-R Baturité está acima de 120. Contudo, alcançar essa meta pode não ser indicativo de qualidade do serviço na rede de atenção à saúde. Estudo realizado no Paraná mostrou que existe pouca integração da ação reabilitadora às ações de promoção e prevenção aos usuários, devendo, portanto, haver um acompanhamento no sistema de referência para a atenção secundária ${ }^{18}$

Mesmo não sendo especialidade mínima prevista para repasse de recursos federais para os CEO, a especialidade de ortodontia teve consultas ofertadas de forma irregular ao longo do ano avaliado, observando-se uma média de vagas abaixo do pactuado. 
Estudo realizado na Bahia apontou como dificuldade para essa especialidade a grande quantidade de retornos mensais para consultas de manutenção, bem como o longo tempo exigido para conclusão dos tratamentos iniciados, e a inoperância do sistema de referência e triagem, são barreiras que tornam complexa a oferta desse tipo de serviço no SUS ${ }^{19}$.

Alguns fatores podem estar relacionados com a disparidade na quantidade de procedimentos realizados durante os meses, tais como: falta de procura dos pacientes, longas filas de espera, ou ainda que o caso tenha se agravado e 0 paciente já necessitaria de outro tipo de atenção ou tratamento.

\section{Considerações Finais}

O CEO-R de Baturité não cumpriu a totalidade de metas previstas na Portaria GM n 1.464/2011 e no contrato de programa do CPS, estando sujeito à suspensão dos repasses de recursos financeiros federais e consorciais. Caso isso aconteça pode comprometer a oferta de serviços e dificultar o acesso da população a atenção especializada em saúde bucal.

Na especialidade de PNE, a meta de procedimentos restauradores não foi cumprida utilizando a portaria como parâmetro. Em relação a oferta de vagas do Contrato de Programa Consorcial, apenas a especialidade de ortodontia não cumpriu com o pactuado, estando sujeito a cobrança dos municípios e gestores que compõe o CPS.

$O$ resultado da avaliação dos serviços no CEO constitui-se uma ferramenta importante no planejamento e no processo de tomada de decisão. Sugere-se a realização de inquéritos epidemiológicos para adequação das metas do Contrato de Programa às necessidades de atenção à saúde bucal, bem como a melhoria da atuação na Rede de Atenção à Saúde Bucal.

O uso de dados secundários a partir dos sistemas de informações em saúde pode ser apontado como limitação para este estudo, pois podem não refletir o quantitativo de procedimentos e atendimentos realizados na prática dos serviços de saúde, ao considerarmos possíveis erros na alimentação dos sistemas de informação. No entanto, o DATASUS é a fonte oficial do Ministério da Saúde e constitui-se como a principal ferramenta para monitoramento e avaliação no SUS.

\section{Referências}

1. Freitas CHSM, LEMOS GA, PESSOA TRRF, ARAUJO MF, FORTE FDS. Atenção em saúde bucal: avaliação dos Centros de Especialidades Odontológicas da Paraíba. Saúde Debate. 2016; 40(108): 131-43.

2. Machado FCA, Silva JV, Ferreira MAF. Fatores relacionados ao desempenho de Centros de Especialidades Odontológicas. Ciência \& Saúde Coletiva. 2015; 20(4):1149-1163.

3. Kornis GEM, Maia LS, Fortuna RFP. Evolução do financiamento da atenção à saúde bucal no SUS: uma análise do processo de reorganização assistencial frente aos incentivos federais. Physis Revista de Saúde Coletiva, Rio de Janeiro. 2011; 21(1):197-215.

4. Laroque MB, Fassa AG, Castilhos ED. Avaliação da atenção secundária em saúde bucal do Centro de Especialidades Odontológicas de Pelotas, Rio Grande do Sul, 2012-2013. Epidemiol. Serv. Saúde, Brasília, jul-set 2015; 24(3):421-30.

5. Brasil. DATASUS - Departamento de Informática do SUS. Ministério da Saúde. [Acesso em 2015 Jun 15]. Disponível em: http://datasus.saude.gov.br/

6. Brasil. Gabinete do Ministro. Portaria n 1.464 , de 24 de junho de 2011. Altera o Anexo da Portaria $n^{0}$ 600/GM/MS, de 23 de março de 2006, que institui o financiamento dos Centros de Especialidades Odontológicas (CEO). Ministério da Saúde, 2011.

7. Brasil. Programa Nacional de Melhoria do Acesso e da Qualidade dos Centros de Especialidades odontológicas (PMAQ-CEO): Manual Instrutivo. Brasília. Ministério da Saúde, 2013.

8. Ceará. Secretaria da Saúde do Estado do Ceará. Relatório de implantação dos Centros de Especialidades Odontológicas Regionais, 2015. [Acesso em 2016 Mai 14]. Disponível em: http://saude.ce.gov.br/

9. Chaves SCL, Soares FF, Rossi TRA, Cangussu MCT, Figueiredo ACL, Cruz DN, et al. Características do acesso e utilização de serviços odontológicos em municípios de médio porte. Ciência \& Saúde Coletiva. 2012; 17(11):3115-24. 
10. Wang H, Northridge ME, Kunzel C, Zhang Q, Kum SS, Gilbert JL, et al. Modeling Social Capital as Dynamic Networks to Promote Access to Oral Healthcare. Soc Cult Behav Model. 2016; 9708: 117-30.

11. Cortellazzi KL, Balbino EC, Guerra LM, Vazquez FL, Bulgareli JV, Ambrosano JMB, et al. Variáveis associadas ao desempenho de Centros de Especialidades Odontológicas no Brasil. Rev Bras Epidemiol. out-dez 2014; 17(4): 978-88. 12. Herkrath FJ, HerkrathAPCQ, Costa LNBS, Gonçalves MJF. Desempenho dos Centros de Especialidades Odontológicas frente ao quadro sociodemográfico dos municípios do Amazonas, Brasil, 2009. Saúde em Debate. jan./mar. 2013; 37(96): $148-58$.

13. Pinto VPT, Teixeira AH, Santos PR, Araújo MWA, Moreira MAG, Saraiva SRM. Avaliação da acessibilidade ao Centro de Especialidades Odontológicas de abrangência macrorregional de Sobral, Ceará, Brasil. Ciência \& Saúde Coletiva, 2014; 19(7):2235-44.

14. Goes PSA. Avaliação da atenção secundária em saúde bucal: uma investigação nos centros de especialidades do Brasil. Cad. Saúde Pública, Rio de Janeiro. 2012;28 Sup:S81-S89.

15. Lino PA, Werneck MAF, Lucas SD, Abreu MHNG. Análise da atenção secundária em saúde bucal no estado de Minas Gerais, Brasil. Ciência \& Saúde Coletiva, 2014;19(9):3879-88.

16. Santana DA, Santos LPS, Carvalho FS, Carvalho CAP. Desempenho de centros de Especialidades Odontológicas de uma Região de Saúde da Bahia. Cad Saúde Colet. 2015; 23(3): 261-7.

17. Filgueira ACC. Avaliação do Desempenho da Política Pública dos Centros de Especialidades Odontológicas na Região do Cariri-CE. Dissertação [Mestrado em Saúde Pública] Universidade Federal do Ceará. Fortaleza; 2012.

18. Possamai T, Pilger $C$, Lentsck MH, Mestriner Junior W. Ações secundárias em saúde bucal: análise do Centro de Especialidades Odontológicas de um município do interior do Paraná, Brasil. Rev. APS. 2015 jul/set; 18(3): 266-72.

19. Souza LF, Chaves SCL. Política Nacional de Saúde Bucal: acessibilidade e utilização de serviços odontológicos especializados em um município de médio porte na Bahia. Rev baiana saúde pública. abr.jun. 2010; 34(2): 371-87.

\section{Paulo Leonardo Ponte Marques}

Endereço para correspondência - Av. Washington Soares, 1321, Edson Queiroz, Cidade: Fortaleza, Estado: Ceará, Brasil CEP: 60811-905.

E-mail: paulomarques@unifor.br

Lattes: http://lattes.cnpq.br/7934153093330220

Antonio Rodrigues Ferreira Junior - junioruruoca@hotmail.com

Raiane Souza Saraiva - raianessaraiva@hotmail.com

Sarenne Pacheco Barbosa Carioca - sarennepbc@hotmail.com

Lucianna Leite Pequeno - luciannapequeno@gmail.com

Luiza Jane Eyre de Souza Vieira - janeeyre@unifor.br

\section{Enviado em 10 de Novembro de 2016. Aceito em 18 de Agosto de 2017.}

